\title{
Extraction of Co(II), Ni(II), Cu(II) and Mn(II) with Deep Eutectic Solvents Dissolved in Heptane as Extractants
}

\author{
Pius Dore OLA ${ }^{1, *}$, Yusuke KUROBE ${ }^{2}$ and Michiaki MATSUMOTO ${ }^{2}$ \\ ${ }^{1}$ Department of Chemistry, Faculty of Science and Engineering, Nusa Cendana University, Jl. Adisucipto \\ 10, Kupang, Indonesia, 85001; ${ }^{2}$ Department of Chemical Engineering and Materials Science, Doshisha \\ University, Kyotanabe, Kyoto 610-0321 Japan
}

(Received March 5, 2021; Accepted May 11, 2021)

Recovering, removing, or concentrating metal ions from wastewater containing metal ions is critical because a negative impact can be generated on living organisms by metal-contained wastewater, which contains high concentration of metals, when it is discarded into the environment without pretreatment. In this work we used deep eutectic solvents (DESs) dissolved in heptane as extractants to separate the metal ions from aqueous media with solvent extraction technique and found that DES composed of lidocaine and decanoic acid was a great extractant with extractability that reached $100 \%$ for interested metal ions. All the metal ions in the experiment were completely stripped out using $1.0 \mathrm{M}$ of hydrochloric acid. DES can be employed as an extractant of metal ions for three extraction cycles with extractability exceeding $90 \%$.

\section{Introduction}

Generally, wastewater produced by the chemical, metallurgical, and mining industries contains valuable metal salt. This contaminated wastewater endangers living organisms. A well-established method for the recovery of metals is solvent extraction [1]. That is due to the following advantages: implementation in a continuous mode, suitability for the processing of high metal feed concentration [2], easy adjustment of the parameters that control the extraction, high efficiency and selectivity, and wide application in metal extraction [3,4]. Organic solvents are commonly used as extractants in the solvent extraction. However, such properties as volatility, flammability, and harmfulness have limited applications in industrial areas. Ionic liquids with such characteristics as green solvent can overcome organic solvent limitations because of such properties as very low vapor pressure, low flammability, and a broad liquid range [5]. However, using ionic liquid as an extractant failed to solve the problems of the solvent extraction technique. Its main problem is the very high cost of ionic liquid caused by the difficulties of its purification. Therefore, creating environmentally friendly extractants remains challenging. Deep eutectic solvent (DES) was developed to overcome the limitations of ionic liquids.

DES is synthesized by mixing two or three inexpensive and safe compounds that act as hydrogen bond donors (HBDs) and hydrogen bond acceptors (HBAs), to form a eutectic mixture because of hydrogen bonding between HBDs and HBAs [6,7]. The melting point of DES is far below the individual components due to large and non-symmetric ions with low lattice energy [8]. Major advantages of DES over ionic liquids include lower prices and easy preparation based on simply mixing the two solid 
constituents at moderate temperatures resulting in a liquid DES, without solvent or complex purification steps. DES have large potential for a wide variety of application, such as metal processing, organic synthesis, biodiesel purification, and catalysis due to their intriguing properties [8]. These DES properties can be used as extractant in the solvent extraction technique as a substitute for ionic liquid because DES overcomes the limitations of ionic liquids and maintains their advantages. One application of DES without diluent was scrutinized as an extractant for the separation of metal ions [9]. Unfortunately, because water was transferred to the DES phase, the volumes of both phases changed considerably from before to after equilibration.

In our preliminary report [10], we found that the DES composed of lidocaine and decanoic acid diluted in heptane successfully functions as the extractant for the quantitative extraction of Fe(III) and $\mathrm{Mn}$ (II) without volume changes of both phases. In this study, we investigated the detailed properties of the DES extractants including the effects of $\mathrm{pH}$ and DES concentration on the extractability, stripping performance, and their reusability for the extraction process of transition metals including manganese(II), cobalt(II), nickel(II), and copper(II).

\section{Experimental}

\subsection{Chemicals}

Decanoic acid, dodecanoic acid, lidocaine, DL-menthol and sodium acetate trihydrate were purchased from Wako Pure Chemicals and the molecular structures of the DES constituents are shown in Figure 1. Manganese chloride, cobalt chloride, nickel chloride, copper chloride, nitric acid and acetic acid were purchased from Nacalai Tesque, Inc., Kyoto. All chemicals were used without further purification.

\subsection{DES Preparation}

DESs were prepared according to the procedure reported in a previous paper [11]: HBD and HBA in a predetermined molar ratio were weighed in a beaker, which was closed with para-film to avoid contamination with water (lidocaine: decanoic acid $=1: 2$, lidocaine: dodecanoic acid $=1: 2$, DL-menthol: decanoic acid $=1: 1$, DL-menthol: dodecanoic acid $=2: 1$ ). The mixture was stirred at $50{ }^{\circ} \mathrm{C}$ until a clear and homogeneous liquid appeared. After that, the DESs were left overnight at room temperature to confirm whether no recrystallization occurred.

\subsection{Solvent extraction of metal ions}

We prepared the DES solution ( $300 \mathrm{~g} / \mathrm{L})$ as an organic phase by dissolving the DES in n-heptane and metal aqueous solution $(10 \mathrm{mmol} / \mathrm{L})$, whose $\mathrm{pH}$ was adjusted by $0.1 \mathrm{~mol} / \mathrm{L}$ of nitric acid, $0.1 \mathrm{~mol} / \mathrm{L}$ of acetic acid, and $0.1 \mathrm{~mol} / \mathrm{L}$ of sodium acetate. Equal volumes $\left(2 \mathrm{~cm}^{3}\right)$ of the aqueous solution and DES solution were mixed and shaken $(120 \mathrm{rpm})$ in a thermostat water bath at $303 \mathrm{~K}$. After shaking 3 hours to attain equilibrium, the concentration of metal in the aqueous solution using inductively coupled plasma atomic emission spectroscopy (ICP-AES, ICPS-8100, Shimadzu, Kyoto, Japan). The $\mathrm{pH}$ values of the aqueous solution were measured with a $\mathrm{pH}$ meter (F-52, Horiba, Kyoto, Japan). Extractability, $\% E$ was calculated using Eq. (1).

$$
\% E=\frac{[\mathrm{M}]_{\mathrm{aq}, \text { int }}-[\mathrm{M}]_{\mathrm{aq}, \mathrm{eq}}}{[\mathrm{M}]_{\mathrm{aq}, \text { int }}} \times 100
$$

where $[\mathrm{M}]$ is the concentration of the metal, aq and org indicate the aqueous and organic phases, and int 
and eq denote the initial and equilibrium states.

\subsection{Stripping of metal ions}

First metal was extracted to the organic solution with DES consisting of lidocaine and decanoic acid under the initial condition of $\mathrm{pH} 5$. A metal-stripping experiment was carried out by mixing a metal-loaded DES solution with hydrochloric acid of various concentrations as a stripping agent. The mixture of equal volumes $\left(2 \mathrm{~cm}^{3}\right)$ of metal-loaded organic solution and hydrochloric acid solution was shaken for 3 hours to allow complete separation between the aqueous and organic phases. We measured the concentration of metals in aqueous phase using ICP-AES. Extent of metal stripping, $\% S$ was calculated using Eq. (2).

$$
\% S=\frac{[\mathrm{M}]_{\mathrm{aq}, \mathrm{eq}}}{[\mathrm{M}]_{\text {org,int }}} \times 100
$$

where $[\mathrm{M}]_{\text {org.int }}$ is the initial concentration of metal in the organic phase, which is the difference in the metal concentration in the aqueous solution before and after it is mixed with the DES solution.

\subsection{Reusability of DES extractant}

We evaluated the extractant reusability using the solvent extraction procedure with the regenerated DES solution produced from the metal-stripping experiment as an extractant. The $\mathrm{Cu}(\mathrm{II})$ in the metal-loaded DES phase was stripped out, and the produced organic phase was used as an extractant. This procedure (initial $\mathrm{pH}=5$ in extraction and $\mathrm{HCl}$ concentration $=5 \mathrm{~mol} / \mathrm{dm}^{3}$ in stripping) was repeated for three cycles.

(a)<smiles>CCCCCCCCCC(=O)O</smiles>

(c)<smiles>CCCCCCCCCCCC(=O)O</smiles>

(b)<smiles>CCN(CC)CC(=O)Nc1c(C)cccc1C</smiles>

(d)

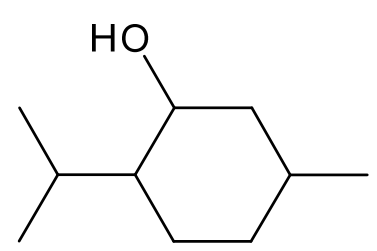

Figure 1. Molecule structures of DES constituents: (a) decanoic acid (b) lidocaine (2-(diethylamino)- $N$-(2,6-dimethylphenyl) acetamide, (c) dodecanoic acid and (d) DL-menthol (5-methyl-2-isopropane) cyclohexane-1-ol)

\section{Results and Discussion}

\subsection{Extraction of metal ions}

We previously reported that a DES composed of lidocaine as HBA and decanoic acid as HBD diluted in heptane functions an extractant for extraction of $\mathrm{Fe}(\mathrm{III})$ and $\mathrm{Mn}$ (II) [10]. In this work, we also used DL-menthol as HBA which has a lesser proton-acceptor compared to lidocaine and dodecanoic acid as HBD with a longer alkyl-chain length than decanoic acid. We combined these HBAs and HBDs to synthesize DESs and employed them for evaluating the effects of HBAs and HBDs on the extractability of 
metal ions. To confirm the performance of DES extractant, we examined the extraction of Mn(II) with one component of DES, lidocaine, DL-menthol or decanoic acid, as shown in Figure 2. The concentrations of DES-constituent components, lidocaine, DL-menthol and decanoic acid were the same as that thet for DES. From Figure 2(a), the extractability of Mn(II) with DES composed of lidocaine and decanoic acid was much higher than that with lidocaine or decanoic acid at higher initial $\mathrm{pH}$, while the extent of enhancement of the extractability with DES composed of DL-menthol and decanoic acid was small. Similar results have been reported for the extraction of riboflavin with same DESs without diluent [12]. Although the reasons for this was not described clearly, higher contents of acids in lidocaine-based DES compared to those in DL-menthol-based DES may have contributed to the higher extractability.
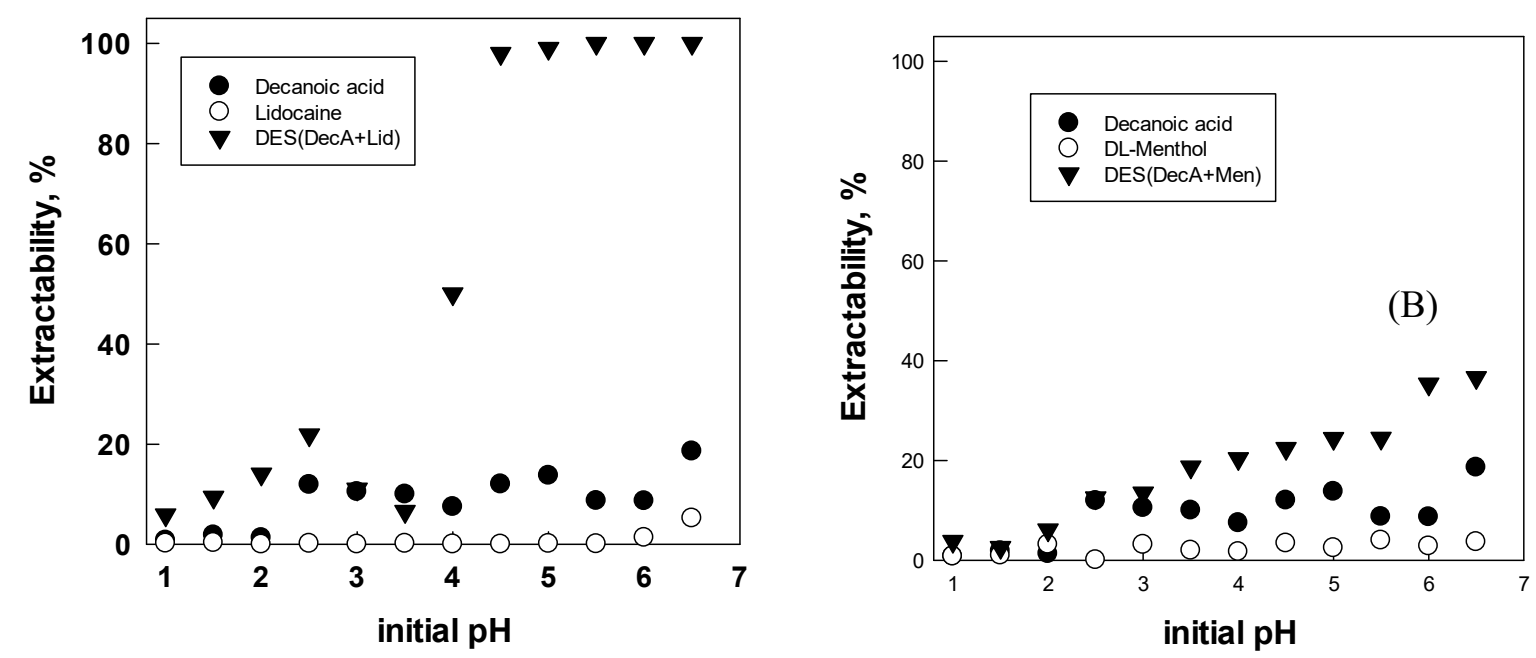

Figure 2. Effect of DES-constituting component on extractability of Mn(II): (A) DES composed of decanoic acid and lidocaine and (B) DES composed decanoic acid and DL-menthol

Figure 3 shows the effect of concentration of DES composed of lidocaine and decanoic acid on the extractability of $\mathrm{Mn}(\mathrm{II})$. The extractability increased with DES concentration and became constant above $300 \mathrm{~g} / \mathrm{L}$. In the following experiments, we used $300 \mathrm{~g} / \mathrm{L}$ of DES concentration.

The $\mathrm{pH}$ effect on the extractability of $\mathrm{Mn}(\mathrm{II}), \mathrm{Ni}(\mathrm{II}), \mathrm{Co}(\mathrm{II})$, and $\mathrm{Cu}(\mathrm{II})$ with DESs is shown in Figure 4. The DES composed of lidocaine and decanoic acid can quantitively extract all metal ions excepting $\mathrm{Mn}(\mathrm{II})$ extraction at low $\mathrm{pH}$. By replacing HBD from decanoic acid to dodecanoic acid, the extractability generally decreased. The higher extractability with DES containing decanoic acid may be caused by the stronger interaction between HBD and HBA due to the lower $\mathrm{p} K_{\mathrm{a}}$ of decanoic acid (4.95) compared to that of dodecanoic acid (5.30). The extractability generally increased with $\mathrm{pH}$, suggesting a proton exchange reaction. As described above, the DES composed of DL-menthol and decanoic acid gave the lower extractability and was only suitable for the extraction of $\mathrm{Cu}(\mathrm{II})$. 


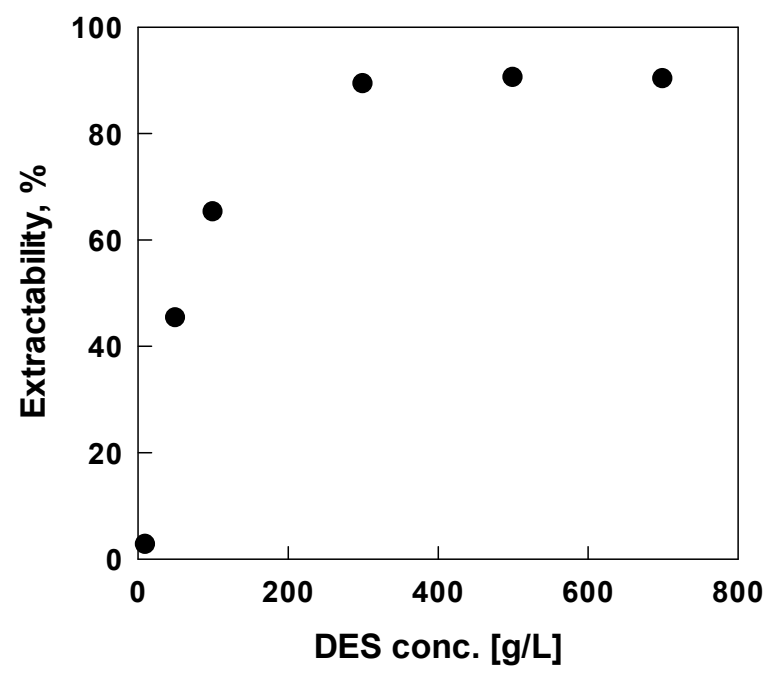

Figure 3. Effect of concentration of DES composed of decanoic acid and lidocaine on extractability of $\mathrm{Mn}(\mathrm{II})$
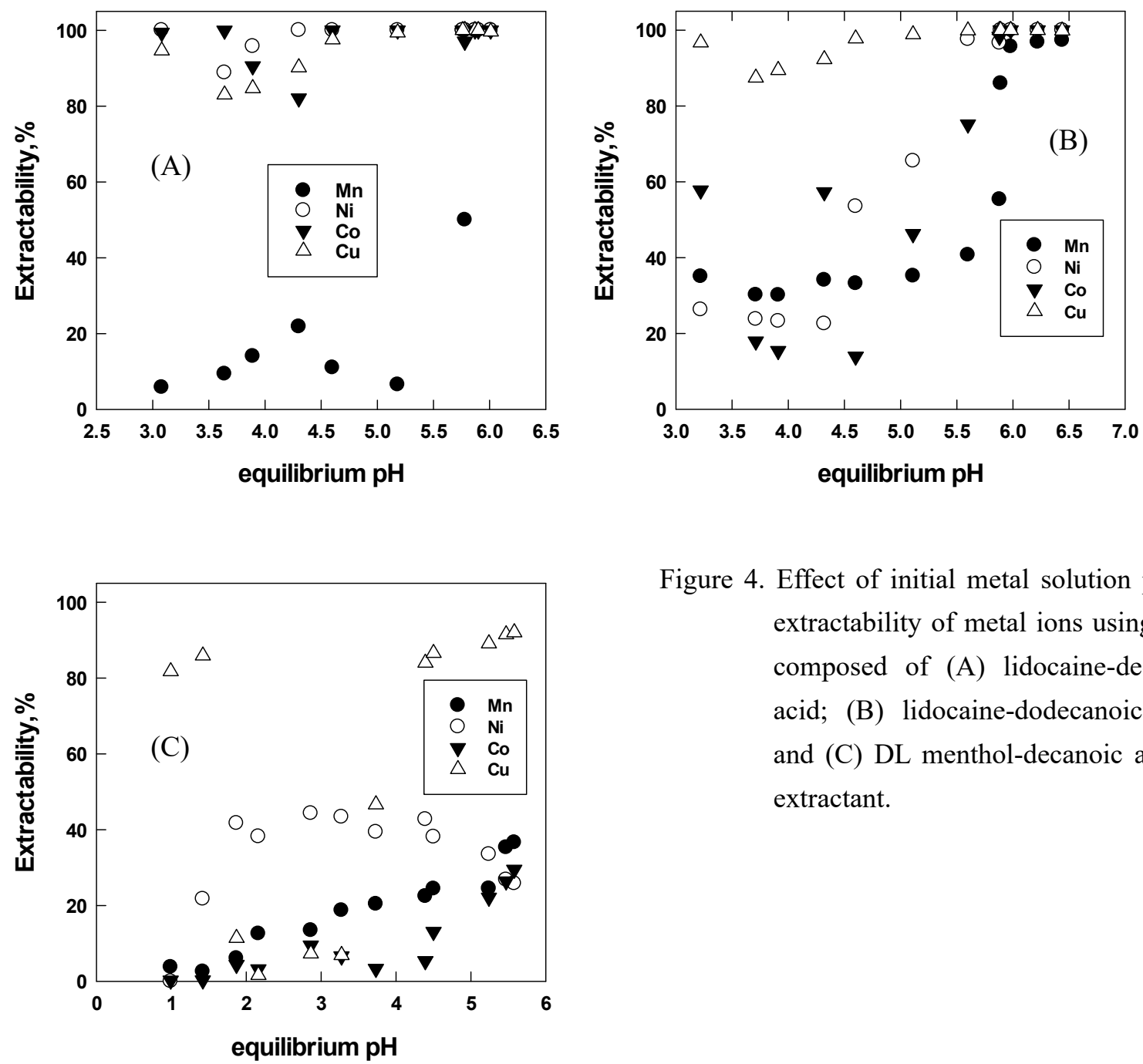

Figure 4. Effect of initial metal solution $\mathrm{pH}$ on extractability of metal ions using DES composed of (A) lidocaine-decanoic acid; (B) lidocaine-dodecanoic acid; and (C) DL menthol-decanoic acid as extractant. 


\subsection{Stripping of metal ions}

Because the DES composed of lidocaine and decanoic acid exhibited higher extractability than the other two DESs, we applied this DES to evaluate metal stripping using hydrochloric acid as a stripping agent. If the metal extraction proceeds by a proton exchange reaction, strong acidic condition will be suitable for stripping, although sodium oxalate as the stripping reagent was used in the same DES extraction system without diluent [9]. Figure 5 shows the effect of concentration of hydrochloric acid on the $\% \mathrm{~S}$. As expected, stripping of the metal ions increased with hydrochloric acid concentration and was almost completed at a hydrochloric acid of $1.0 \mathrm{~mol} / \mathrm{L}$. Furthermore, Mn(II) stripping was slightly higher than the others because the extractability of $\mathrm{Mn}$ (II) was lower than the other metals. This means that the higher the extractability of metal ions, the lower its stripping percentage.

From the extraction and stripping experiments, we speculated the extraction reaction is proceeded by a proton exchange reaction as shown by Eq. (3).

$$
\mathrm{Mn}_{\mathrm{aq}}^{2+}+2 \mathrm{RCOOH}_{\mathrm{org}}+\mathrm{nLid}_{\mathrm{org}} \rightleftharpoons \mathrm{Mn}(\mathrm{RCOO})_{2}(\mathrm{Lid})_{\mathrm{n} \mathrm{org}}+2 \mathrm{H}_{\mathrm{aq}}^{+}
$$

In the future study, we will clarify the detailed extraction mechanism by examining the concentration dependency of concerned species.

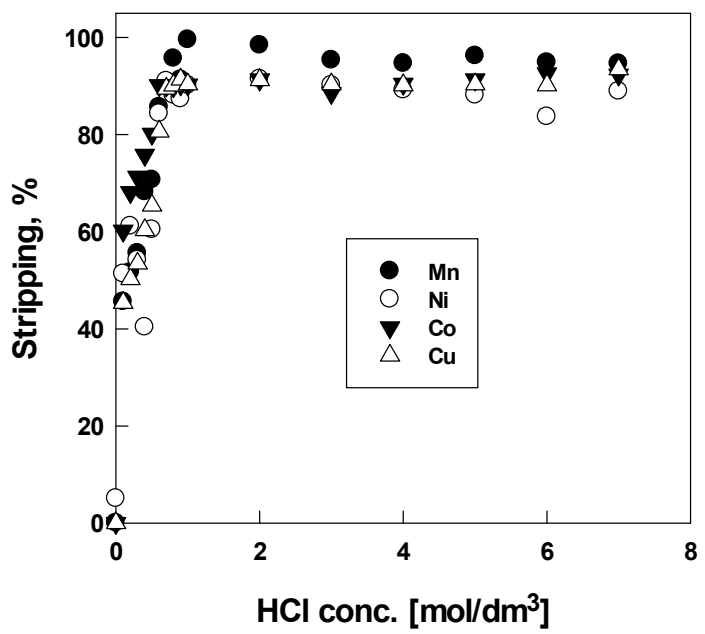

Figure 5. Effect of hydrochloric acid concentration on stripping of metal ions from metal-loaded DES solution

\subsection{Reusability of DES extractant}

To evaluate the DES extractant reusability, we used a regenerated DES solution in the copper extraction after the stripping from $\mathrm{Cu}(\mathrm{II})$-loaded solution. Table 1 shows the experimental results. The extractability of $\mathrm{Cu}(\mathrm{II})$ remained $>90 \%$ for each DES extractant until the three cycles. 
Table 1. Reusability of DES for extraction of $\mathrm{Cu}(\mathrm{II})$

\begin{tabular}{rcc}
\hline \multirow{2}{*}{ Cycle } & \multicolumn{2}{c}{ Extractability, \% } \\
\cline { 2 - 3 } & DL menthol-Decanoic acid & Lidocaine-Decanoic acid \\
\hline 1 & 95.7 & 98.7 \\
2 & 94.4 & 97.7 \\
3 & 90.4 & 96.2 \\
\hline
\end{tabular}

\section{Conclusion}

We examined the extraction of $\mathrm{Mn}(\mathrm{II}), \mathrm{Cu}(\mathrm{II}), \mathrm{Co}(\mathrm{II})$ and $\mathrm{Ni}(\mathrm{II})$ using DESs as extractants. We found that a DES composed of lidocaine-decanoic acid was the best extractant followed by a DES composed of lidocaine-dodecanoic acid. A DES composed of DL menthol-decanoic acid was only suitable for the extraction of $\mathrm{Cu}(\mathrm{II})$. All the metal ions in our experiments were stripped from the metal-loaded DES phase using $1.0 \mathrm{~mol} / \mathrm{L}$ of hydrochloric acid. From the extraction and stripping experiments, we speculated the extraction reaction by a proton exchange reaction. Our evaluation of DES reusability showed that it retains the high extractability of $\mathrm{Cu}$ (II) ions for three extraction cycles.

\section{References}

1) T. Miaomiao, J. Quang, L. Wuping, J. Rare Earths, 31, 604-608 (2013).

2) T. V. Hoogerstraete, S. Wellens, K. Verachtert, K. Binnemans, Green Chem., 15, 919-927 (2013).

3) Y. Baba, F. Kubota, N. Kamiya, M. Goto, J. Chem. Eng. Jpn., 44, 679-685 (2011).

4) A. P. de Los Ríos, F. J. Hernández-Fernández, L. J. Lozano, S. Sánchez, J. I. Moreno, C. Godínez , J. Chem. Eng. Data, 55, 605-608 (2010).

5) M. Matsumoto, Y. Inomoto, K. Kondo, J. Membr. Sci., 26, 77-81 (2005).

6) B. Y. Zhao, P. Xu, F. X. Yang, H. Wu, M. H. Zong, W. Y. Lou, ACS Sustainable Chem. Eng., 3, 2746-2755 (2015).

7) Q. Zhang, K. D. O. Vigier, S. Royer, F. Jérôme, Chem. Soc. Rev., 41, $7108-7146$ (2012).

8) E. L. Smith, A. P. Abbott, K. S. Ryder, Chem. Rev., 114, 11060-11082 (2014).

9) D. J. G. P. van Osch, D. Parmentier, C. H. J. T. Dietz, A. van den Bruinhorst, R. Tuinier, M. C. Kroon Chem. Commun., 52, 11987-11990 (2016).

10) P. D. Ola, M. Matsumoto, Sep. Sci. Technol., 54, 759-765 (2019).

11) P. J. Griffin, T. Cosby, A. P. Holt, R. S. Benson, J. R. Sangoro, J. Phys. Chem. B, 118, 9378-9385 (2014).

12) D. J. G. P. van Osch, C. H. J. T. Dietz, J. van Spronsen, M. C. Kroon, F. Gallucci, M. van Sint Annaland, R. Tuinier, ACS Sustainable Chem. Eng., 7, 2933-2942 (2019). 